\title{
Caracterização de Lipídios de Sementes de Canola na Região Dos Campos Gerais, Paraná $^{1}$
}

Alison Andrei Schmatz ${ }^{2}$, Lucas Henrique Waiga ${ }^{3}$, Maria Elena Payret Arrua ${ }^{4}$, Sandra Regina Masetto Antunes ${ }^{4}$, Pedro Henrique Werich Neto ${ }^{4}$, David de Souza Jaccoud Filho ${ }^{5}$, Ayrton Berger Neto ${ }^{6}$, Augusto Celso Antunes ${ }^{4}$

${ }^{1}$ Aceito para publicação no $3^{\circ}$ Trimestre de 2014

2 Mestrando em Bioenergia na Universidade Estadual de Ponta Grossa, alison_schmatz@hotmail.com

${ }^{3}$ Acadêmico em Química na Universidade Estadual de Ponta Grossa, lwaiga@ hotmail.com

${ }^{4}$ Professores do Programa de Pós- Graduação em Bioenergia na Universidade Estadual de Ponta Grossa, elenapayret@gmail.com; sandrareg@uepg.br; lama1@uepg.br; ac_antunes.com.br

${ }^{5}$ Professor do Departamento de Fitotecnia e Fitossanidade na Universidade Estadual de Ponta Grossa, di1002@uepg.br

6 Mestrando em Agronomia na Universidade Estadual de Ponta Grossa, neto_berguer@hotmail.com

\section{RESUMO}

Uma grande variedade de oleaginosas pode ser utilizada como matéria prima na produção de biodiesel, como soja, algodão e canola entre outras. A Canola (Brassica napus) é uma espécie de oleaginosa que apresenta alto teor de lipídios, sendo largamente utilizada para esse fim em alguns países da Europa. Este trabalho teve como objetivo caracterizar os lipídios de sementes de canola cultivada em Tibagi, região dos Campos Gerais, visando uso na produção de biodiesel. Foram utilizadas sementes colhidas em lavoura comercial da safra de inverno de 2013. O sistema de extração de óleo utilizado foi extração contínua por solvente. As propriedades dos lipídios obtidos foram determinadas com relação à massa relativa de lipídios, massa específica e estabilidade oxidativa, que tiveram média de $370,4 \mathrm{~g} \mathrm{~kg}^{-1}$, $873 \mathrm{~kg} \mathrm{~m}^{-3}$, e 10,01 horas, respectivamente. A composição qualitativa de ácidos graxos foi avaliada pela Espectroscopia de Ressonância Magnética Nuclear de ${ }^{1} \mathrm{H}$. Pelas análises foi constatada a presença de ácidos Oleico (C18:1), Linoléico (C18:2) e Linolênico (C18:3).

PALAVRAS-CHAVE: biodiesel, óleo vegetal, bioenergia 


\begin{abstract}
A great variety of oil seed grains can be used as raw material to produce biodiesel, for example: soybean, cotton and canola among others. Canola (Brassica napus) is one of the oil seed species with high lipids content in its grains, and is largely employed with this purpose in some European countries. Therefore, the objective of this study was to characterize Canola seed oil lipids ((Brassica napus)), planted in Tibagi, region of Campos Gerais, aiming to produce biodiesel. Seeds picked in a commercial crop in the winter 2013 were used. The oil extraction system used was solvent. The properties of the lipids obtained were determined in relation to lipids relative mass, specific mass and oxidative stability, which presented $370,4 \mathrm{~g}$ $\mathrm{kg}^{-1}, 873 \mathrm{kgm}^{-3}$ and 10,01 hour average, respectively. Fatty acid composition was assessed through the Nuclear Magnetic Resonance Spectroscopy ${ }^{1} \mathrm{H}$. The NMR analysis revealed the presence of oleic (C18:1), linoleic (C18:2) and linolenic (C18:3) acids.
\end{abstract}

KEYWORDS: biodiesel, vegetable oil, bioenergy

\title{
INTRODUÇÃO
}

Os biocombustíveis representam potencial fonte de energia renovável, que podem gerar novos e grandes mercados para a agricultura brasileira. Várias matérias primas podem ser utilizadas para este fim, como a cana de açúcar e o milho na produção de etanol e, várias oleaginosas na produção de biodiesel. Entre as oleaginosas mais utilizadas encontram-se a soja, o girassol, o amendoim, o algodão, e a canola (ALBUQUERQUE, 2006).

A canola, Brassica napus, é uma oleaginosa de clima temperado e regiões com latitudes entre 35 a 55 graus. Pertencente ao gênero Brassica da família Brassicaceae, seus grãos possuem em torno de $38 \%$ de lipídios, e como subproduto da extração do mesmo, obtém-se o farelo de canola com $34 \%$ a $38 \%$ de proteínas que pode ser utilizado na formulação de rações para bovinos, suínos, ovinos e aves (BORSOI, 2012). A canola é uma variedade obtida a partir do melhoramento genético da colza, que teve seu nome derivado de CANadian Oil, Low Acid, criada em 1974 pela Universidade de Manitoba, Canadá (ALBUQUERQUE, 2006).

A canola é a terceira oleaginosa mais produzida no mundo, e seu óleo apresenta alto percentual de gorduras insaturadas, cerca de 90\%. O principal ácido graxo encontrado no óleo de canola é o ácido oléico, também conhecido como Omega-9, em torno de 70\% (PANOZZO, 2012). 
As condições climáticas, relacionadas com localização, altitude, e ano agrícola são de suma importância par o manejo das culturas agrícolas bem como para rendimentos quantitativos e qualitativos. Fatores ambientais como temperatura do solo e do ar, radiação solar e condições hídricas exercem influência direta no desenvolvimento da cultura, podendo afetar também a qualidade das sementes e, portanto, as características dos constituintes do óleo (TOMM, 2007).

Cada tipo de oleaginosa poderá dar origem a lipídios com características únicas e diferentes, como o perfil de ácidos graxos, essa peculiaridade gera diferença significativa quando os óleos são empregados na produção de biodiesel, gerando assim, combustíveis com diferentes propriedades e implicações em sua qualidade (PINZI et al., 2011 e PANOZZO, 2012).

Estudando dois híbridos de canola em diferentes épocas de semeadura na região oeste do estado do Paraná, Melgarejo et al. (2014) verificaram diferentes rendimentos de massa de sementes e de massa relativa de óleos para as épocas de semeadura. O referido trabalho mostra que, em épocas com restrições pluviométricas houve diminuição no rendimento de massa de sementes e de massa relativa de óleos. Em trabalhos com soja com diferentes épocas de semeadura, na região sul de Minas Gerais, Lélis et al. (2010) observaram interação entre a época de semeadura da planta e o genótipo cultivado, para rendimento de sementes e massa relativa de óleos.

Independentemente da forma de obtenção ou utilização dos lipídios vegetais, o conhecimento de parâmetros como massa especifíca e estabilidade oxidativa são fundamentais para a especificação do produto. Através da mensuração de diferentes óleos vegetais comestíveis, observou-se que não há uma diferença significativa no valor do massa específica em relação à matriz oleaginosa, sendo que a canola a $25 \pm 1{ }^{\circ} \mathrm{C}$ apresentou massa especifíca de 0,878 $\pm \sigma 0,002 \mathrm{~g} \mathrm{~cm}^{-3}$ (BROCK et al., 2008).

A estabilidade oxidativa, de lipídios de origem vegetal e animal, serve como um parâmetro global para avaliação da qualidade da matéria prima para produção de biodiesel. Os métodos de determinação da estabilidade oxidativa surgiram numa tentativa de predizer a vida-de-prateleira do material lipídico, sendo este submetido a processo de oxidação acelerada, sob condições controladas de temperatura e fluxo de ar (ANTONIASSI, 2001).

Para tanto, este trabalho teve como objetivo caracterizar os lipídios de sementes de Canola (Brassica napus), cultivadas na cidade de Tibagi, região dos Campos Gerais, visando uso na produção de biodiesel. 


\section{MATERIAIS E MÉTODOS}

Para os estudos foram utilizadas sementes de canola do híbrido Hyola 43, semeado em quatro épocas diferentes, de 4 de abril a 14 de maio de 2013, utilizando-se três repetições para cada época. Sendo assim foram colhidas 12 amostras de aproximadamente $500 \mathrm{~g}$ cada.

No momento da semeadura utilizou-se 18 sementes por metro com aplicação no sulco de $100 \mathrm{~kg} \mathrm{ha}^{-1}$ de fertilizante (fórmula NPK 13-33-00). Aos quarenta e cinco dias após semeadura foram realizadas aplicações, em área total, de $100 \mathrm{~kg} \mathrm{ha}^{-1}$ uréia e $100 \mathrm{~kg} \mathrm{ha}^{-1} \mathrm{de}$ $\mathrm{KCl}$. Durante o ciclo da cultura não foram aplicados fungicidas, inseticidas e herbicidas. A semeadura foi em propriedade comercial no município de Tibagi, Paraná.

As sementes de canola foram inicialmente peneiradas, com finalidade de separar impurezas dos grãos, como casca, material mineral e outros vestígios de material vegetal proveniente da colheita, que pudessem interferir na composição química do óleo bruto. Posteriormente foram trituras e submetidas à extração contínua em aparelho Soxhlet, utilizando-se $n$-hexano como solvente (AOAC,1995). O extrato hexânico foi filtrado e evaporado. Na sequência, os lipídios obtidos passaram por processo de degomagem aquosa, sendo adicionados 5\% de massa de água em relação à massa total dos lipídios. A mistura permaneceu por 30 minutos sob agitação e temperatura constante de $65^{\circ} \mathrm{C}$. Esse processo tem a finalidade de remover fosfatídeos, dentre outras substâncias coloidais (CORREIA, 2009).

Após a extração foi determinada a massa especifíca dos lipídios, a $20^{\circ} \mathrm{C}$, utilizando densímetro digital Anton Paar, modelo DMA 4500 M.

A estabilidade oxidativa foi determinada em equipamento Rancimat Metrohm, modelo 893. Os lipídios foram avaliados em ensaio de oxidação acelerada, sob condições padronizadas de temperatura e fluxo de $\operatorname{ar}, 110^{\circ} \mathrm{C} \mathrm{e} 10 \mathrm{~L} \mathrm{~h}^{-1}$, respectivamente (ANTONIASSI, 2001).

Os ensaios de determinação da massa específica e da estabilidade oxidativa foram realizados em triplicata (36 análises de cada variável), enquanto a massa de lipídios foi realizada em única repetição para cada amostra (12 análises).

Além disso, as amostras foram caracterizadas por Espectroscopia de RMN de ${ }^{1} \mathrm{H}$, em equipamento BRUKER ASCEND, $400 \mathrm{MHz}$.

\section{RESULTADOS E DISCUSSÃO}

Em análise visual dos lipídios obtidos, considerando-se à coloração e impurezas presentes, observou-se óleo límpido e isento de impurezas. Entretanto, foram observadas diferenças na coloração passando pelo verde/castanho, bem como diferentes tonalidades de 
amarelo. Estas diferenças de coloração, provavelmente, se devam à presença de carotenóides e clorofila (ALI et al., 2009).

$\mathrm{Na}$ tabela 1 está representada a média da massa relativa de lipídios obtidos, bem como valor máximo e mínimo da mesma. Neste caso destaca-se a grande amplitude encontrada, de aproximadamente $130 \mathrm{~g} \mathrm{~kg}^{-1}$, o que pode levar a novos estudos.

Tabela 1: Conteúdo de lipídios mínimo, máximo e média, extraídos de sementes de canola em aparelho Soxhlet, com n-hexano

\begin{tabular}{lc}
\hline \multicolumn{2}{l}{ Massa relativa de lipídios $\left(\mathrm{g} \mathrm{kg}^{-1}\right)$} \\
\hline Valor mínimo & 305,7 \\
Valor máximo & 439,5 \\
Valor médio & 370,4 \\
\hline
\end{tabular}

Segundo Tomm (2007), o valor médio de lipídios esperado no Brasil para sementes de canola é de $380 \mathrm{~g} \mathrm{~kg}^{-1}$. Em experimentos realizados na cidade de Marechal Candido Rondon, Paraná, com diferentes datas de semeadura, Melgarejo et al. (2014) obtiveram valores de massa relativa de lipídios variando de 346 a $399 \mathrm{~g} \mathrm{~kg}^{-1}$.

Na Tabela 2 encontram-se o valor máximo, mínimo, e a média da densidade dos lipídios de sementes de canola.

Tabela 2: Valor mínimo, máximo e médio de massa específica de lipídios de canola (kg m-3) obtidas a $20^{\circ} \mathrm{C}$ em densímetro digital (ensaios realizados em triplicata)

\begin{tabular}{lc}
\hline \multicolumn{2}{c}{ Massa específica a $20^{\circ} \mathrm{C}\left(\mathrm{kg} \mathrm{m}^{-3}\right)$} \\
\hline Valor mínimo & $850 \pm 1,52$ \\
Valor máximo & $905 \pm 2,08$ \\
Valor médio & $873 \pm 1,75$ \\
\hline
\end{tabular}

Os valores de massa específica são comparáveis aos da literatura. Segundo Brock et al. (2008), o valor de massa específica de lipídios de canola é de $878 \mathrm{~kg} \mathrm{~m}^{-3} \pm 0,002$. Ao 
analisar os lipídios vegetais em diversas temperaturas, Esteban et al. (2012) encontraram valor médio de $914,5 \mathrm{~kg} \mathrm{~m}^{-3}$ para massa especifica, a $20{ }^{\circ} \mathrm{C}$, dos lipídios de canola.

A estabilidade oxidativa de lipídios está diretamente relacionada à estrutura das cadeias dos ácidos graxos presentes nos mesmos. Sendo mais estáveis aqueles que apresentam, na sua composição, maior percentagem de ácidos graxos saturados, portanto, podendo ser estocados por mais tempo. Lee et al. (2010), cita que a os principais ácidos graxos encontrados na canola são o oléico $\left(\mathrm{C}_{18: 1}\right)$ com $62 \%$; ácido linoléico $\left(\mathrm{C}_{18: 2}\right)$ com $22 \%$; ácido linolênico $\left(\mathrm{C}_{18: 3}\right)$ com $10 \%$; ácido palmítico $\left(\mathrm{C}_{16: 0}\right)$ com $4 \%$; e ácido esteárico $\left(\mathrm{C}_{18: 0}\right)$ com 2\%. O monitoramento do processo oxidativo das amostras de lipídios foi realizado determinando-se o tempo de indução através do método Rancimat, os resultados são apresentados na Tabela 3.

A estabilidade oxidativa das doze amostras analisadas foi em média de 10,01 horas, este valor está de acordo com os valores indicados pela literatura. Antoniassi (2001) encontrou valores para a estabilidade oxidativa de lipídios de canola de 9,4 a 10,8 horas. A razão dessa baixa estabilidade oxidativa pode ser explicada pela quantidade de ácidos graxos insaturados encontrados na amostra (GHARBY et al., 2014). Os óleos vegetais compostos por ácidos graxos que apresentam maior número de insaturações são mais propensos aos processos oxidativos (KNOTHE, 2006).

Tabela 3: Tempo mínimo, máximo e médio de indução de lipídios de sementes de canola (ensaios realizados em triplicata)

\begin{tabular}{lr}
\hline \multicolumn{2}{l}{ Tempo médio de indução $(\mathrm{h})$} \\
\hline Valor mínimo & $9,00 \pm 0,10$ \\
Valor máximo & $10,98 \pm 0,04$ \\
Valor médio & $10,01 \pm 0,91$ \\
\hline
\end{tabular}

A composição de ácidos graxos foi avaliada por Espectroscopia de RMN de ${ }^{1} \mathrm{H}$. Pelas análises podem-se constatar os sinais referentes aos hidrogênios dos ácidos oléico, linoléico e linolênico (Figura 2).

Pela análise do espectro de RMN de ${ }^{1} \mathrm{H}$ podem ser observados sinais correspondentes aos triacilglicerídeos, sendo que, as cadeias preponderantemente encontradas correspondem 
aos ácidos Oleico (C18:1), Linoléico (18:2) e Linolênico (18:3). No Espectro da Figura 2 observa-se um multipleto na região de $\delta 5,39-5,28$ ppm correspondente aos hidrogênios $\mathrm{H}$ vinílicos presentes nas cadeias do óleo. Já na região de $\delta$ 5,30 - 5,25 ppm observa-se outro multipleto correspondente ao $\mathrm{H}$ denominado como glicerólico. A presença dos hidrogênios $\alpha$ acilicos pode ser observada como um conjunto de sinais característicos (duplos dupletos, dd) em $\delta$ 4,31- 4,12 ppm (JIN et al., 2007). Na região de $\delta$ 2,8-2,7 ppm observou-se um multipleto correspondente aos hidrogênios bis-vinílicos presentes somente no ácido Linolênico. Os hidrogênios $H$ ( $\alpha$-carbonílicos) podem ser localizados em $\delta 2,3$ ppm como tripleto. No intervalo de $\delta$ 2,05 - 2,00 ppm o espectro apresenta um multipleto correspondente aos hidrogênios alílicos. Os sinais de hidrogênios metilênicos podem ser observados como multipleto na faixa de $\delta 1,30-1,26$ ppm e o sinal dos hidrogênios metílicos em $\delta 0,99$ ppm como tripleto.

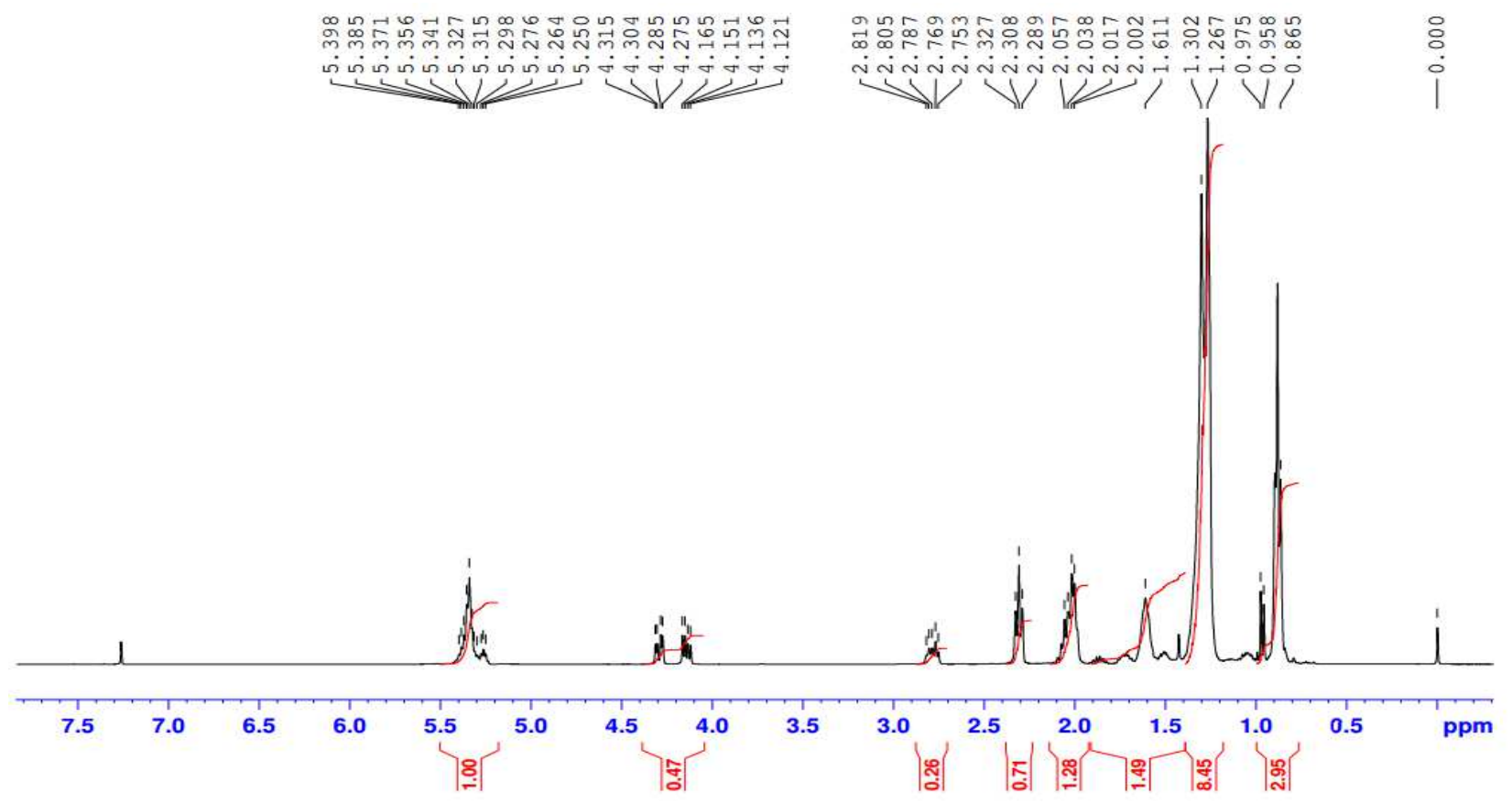

Figura 2: Espectro de RMN de ${ }^{1} \mathrm{H}(400 \mathrm{MHz})$ de amostra de lipídios de canola 


\section{CONCLUSÕES}

A massa relativa de lipídios variou de 305 a $439 \mathrm{~g} \mathrm{~kg}^{-1}$, com média de $370,4 \mathrm{~g} \mathrm{~kg}^{-1} \mathrm{e}$ a massa especifíca de 850 a $905 \mathrm{~kg} \mathrm{~m}^{-3}$, com média de $873 \mathrm{~kg} \mathrm{~m}^{-3}$.

O tempo de indução da estabilidade oxidativa dos lipídios variou de 9,00 a 10,98 horas, com média de 10,01 horas.

Pelas análises de RMN de ${ }^{1} \mathrm{H}$ foi constatada a presença dos ácidos Oleico $\left(\mathrm{C}_{18: 1}\right)$, Linoléico $\left(\mathrm{C}_{18: 2}\right)$ e Linolênico $\left(\mathrm{C}_{18: 3}\right)$ como esperado.

\section{AGRADECIMENTOS}

A Coordenação de Aperfeiçoamento de Pessoal de Nível Superior (CAPES) pela concessão de bolsa de mestrado para o primeiro autor, a Fundação Araucária e Complexo Laboratorial Multiusuário (C-LABMU/UEPG).

\section{REFERÊNCIAS}

ABDUllAH, A. S. Minimum Tillage And Residue Management Increase Soil Water Content, Soil Organic Matter and Canola Seed Yield and Seed Oil Content In The Semiarid Areas Of Northern Iraq. Soil e Tillage Research, 2014.

ALBUQUERQUE, G., A. Obtenção e Caracterização Físico-Química do Biodiesel de Canola. Dissertação de Mestrado, Programa de Pós-Graduação em Química, Universidade Federal da Paraíba, João Pessoa - PB, 2006.

ALI, S. et al., Evaluation of Canola Seeds of different Cultivars With Special Emphasis on the Quantification of Erucic acid and Glucosinolates. GRASAS Y ACEITES, 2009.

ALMEIDA, P. R. Monteiro Lobato e a Emergência da Política do Petróleo no Brasil. Capítulo de colaboração ao livro: Omar L. de Barros Filho e Sylvia Bojunga. 2008.

ANTONIASSI, R. Métodos de avaliação da estabilidade oxidativa de óleos e gorduras. Boletim do Cento de Pesquisa e Processamento de Alimentos, Curitiba, volume 19, n², páginas 353-380. 2001.

AOAC, Cunnif, P., 1995. Official Methods of Analysis of AOAC International, 16th ed. AOAC International, Arlington, VA.

A. MElgarejo, M. A. et. al., Características Agronômicas e teor de Óleo de Canola em Função da Época de Semeadura. Revista

Brasileira de Engenharia Agrícola e Ambiental. Campina Grande, Paraíba, 2014. 
BORSOI, A. Análise Econômica da Produção de Biodiesel para a Agricultura Familiar no Município de Corbélia - PR. Dissertação de mestrado. Programa de Pós-Graduação em Energia na Agricultura, Universidade Estadual do Oeste do Paraná, Cascavel - PR. 2012.

BROCK, L. et al., Determinação Experimental da Viscosidade e Condutividade Térmica de Óleos Vegetais. Ciência e Tecnologia de Alimentos, Campinas, São Paulo, 2008.

BYRDWELL, W. C., NEFF, W. E. Autoxidation products of normal and genetically modified canola oil varieties determined using liquid chromatography with mass spectrometric detection. Journal of Chromatography A, 2001.

CORREIA, I. M. S. Extração e Pirólise do óleo de Girassol (Helianthus annus L.) Visando a Produção de Combustíveis.Dissertação de Mestrado, Universidade Federal do Rio Grande do Norte. Centro de Tecnologia - Departamento de Engenharia Química . Programa de PósGraduação em Engenharia Química. Natal, Rio Grande do Norte, 2009.

ESTEBAN, B. Temperature Dependence Of Density And Viscosity Of Vegetable Oils. Biomass and Bioenergy. 2012.

GARCIA, C. M. Transesterificação de óleos vegetais, Dissertação de Mestrado. Departamento de Química Inorgânica. Universidade Estadual de Campinas, Instituto de Químca, março de 2006.

GHARBY, S. et al., The Stability of Vegetable oils (Sunflower, Rapseed and Palm) Soldo n the Moroccan Market at High Temperature. International Journal of Chemical and Biochemical Sciences, Marrocos, 2014.

JIN, F. et al. NMR spectroscopic Study on Methanolysis Reaction of Vegetable Oil. Fuel. V86, P 1201-1207, 2007.

KNOTHE, G.; GERPEN, J. V.; KRAHL, J.; RAMOS, L.P., Manual do Biodiesel. Traduzido do original "The Biodiesel Handbook"por Luiz Pereira Ramos, São Paulo: Edgard Blucher, 2006.

LEE, S. B. et al. Optimum process and energy density analysis of canola oil biodiesel synthesis. Journal of Industrial and Engineering Chemistry. 2010.

LÉLIS, M. M. et al., Teor De Óleo Para Genótipos De Soja Em Três Épocas De Semeadura. Bioscience Journal. Uberlândia, 2010.

MAZZOLA, A. A. Ressonância Magnética: Princípios da Formação da Imagem e Aplicações em Imagem Funcional. Revista Brasileira de Física Médica. Artigo de Revisão. Porto Alegre, 2009.

PANOZZO, L. E. Qualidade de Sementes, Características Agronômicas e Produtivas de Híbridos de Canola em Diferentes Épocas de Semeadura e Colheita em Viçosa - MG. Tese de 
doutorado, programa de pós-graduação em Fitotecnia, Universidade Federal de Viçosa, Viçosa - MG 2012.

PAULILLO, L. F. et. al., Álcool, combustível e biodiesel no Brasil: Quo Vadis? Revista de Economia e Sociologia Rural. Volume 45, n 3. Brasília, DF. 2007.

PINZI S, Gandia LM, Arzamendi G, Ruiz JJ, Dorado MP. Influence of vegetable oils fatty acid composition on reaction temperature and glycerides conversion to biodiesel during transesterification. Bioresour Technology. 2011

TOMM, G. O. Situação Atual e Perspectivas da Canola no Brasil. EMBRAPA Trigo. Comunicado Técnico on-line, n $^{\circ}$ 58. Passo Fundo - RS. 2000.

TOMM, G. O. Situação em 2005 e Perspectivas da cultura da Canola no Brasil e Países Vizinhos. EMBRAPA Trigo, Boletim de Pesquisa e Desenvolvimento on-line, $\mathrm{n}^{\circ}$ 26. Passo Fundo - RS. 2005.

TOMM, G. O. Indicativos tecnológicos para produção de canola no Rio Grande do Sul. Passo Fundo: Embrapa Trigo, 2007. 32 p.

ZONIN, V.J. et. al. Utilização da Canola Como Alternativa na Cadeia de Suprimentos do Biodiesel: Estudo de Caso em Duas Empresas do RS. $48^{\circ}$ Congresso da Sociedade Brasileira de Economia, Administração e Sociologia Rural. Campo Grande - MS. 2010. 